\title{
FURTHER EVIDENCE OF AN INCOMPATIBILITY ALLELE SYSTEM IN THE COMPLEX-HETEROZYGOTES OF OENOTHERA ${ }^{1}$
}

\author{
Erich Steiner
}

Although SELF-incompatibility in the genus Oenothera is perhaps best known from the work of Emerson (1938, 1939) and of Lewis (1947, 1948, $1949,1951)$ on Oenothera organensis, there are at least eight other species which exhibit the same behavior, as the recent survey of Crowe (1956) has shown. Of the self-fertile species many are translocation heterozygotes which maintain heterozygosis by means of a balanced lethal system. Recently, the writer (Steiner, 1956) has presented evidence that at least one phylogenetic group of such translocation heterozygotes possesses a self-incompatibility system of the oppositional allele type which functions as a part of the balanced lethal mechanism. The present paper is a brief report of additional data which support this hypothesis.

Evidence of an incompatibility allele mechanism has been discovered in oenotheras of the biennis group 1, a phylogenetic grouping characteristic of the midwestern and southeastern United States. Members of the biennis group 1 are translocation heterozygotes composed of two genomes or "complexes" of seven chromosomes each. The two complexes, called alpha and beta, respectively, differ completely in the arrangement of their chromosome segments. Thus at meiosis a circle of fourteen chromosomes is formed and only two kinds of spores are produced, one carrying the alpha complex, the other bearing the beta complex. Among the progeny resulting from self-pollination of such a plant, no homoyzgotes (alpha-alpha or beta.beta) are obtained, and only alphabbeta combinations like the parent occur. Failure of homozygotes to appear is attributed to a balanced lethal system.

In the biennis group 1 , the alpha complexes all produce the biennis phenotype, while the betas carry strigosa characters. Furthermore, the alpha complexes in this group possess, with a few exceptions, the same chromosomal end arrangement, namely, 1.2 3.4 5.14 7.10 9.8 $11 \cdot 12$ 13.6.

Characteristically, in outcrosses the alpha complex is transmitted to the offspring through the egg, while the beta comes through the pollen; this is not a hard and fast distinction, however, since in outcrosses with many of the biennis group 1 races the alpha complex may be transmitted with considerable frequency through the pollen, and the beta on occasion may come through the egg. Therefore, in crossing different races of the biennis group 1 , it is possible to obtain hybrids which carry the alpha complex from each parent. Since the alpha com-

\footnotetext{
${ }^{1}$ Received for publication February 20, 1957.
}

Work supported by the Faculty Research Fund, Horace H. Rackham School of Graduate Studies, University of Michigan. plexes usually possess the same chromosomal end arrangement, alpharalpha hybrids show at meiosis seven pairs of chromosomes. They are thus readily identified cytologically; phenotypically they are also distinctive because of the double dose of biennis characters which they carry.

As reported previously (Steiner, 1956), alpha. alpha hybrids are uniformly self-incompatible, regardless of the biennis group 1 races which have been used as parents. The self-incompatibility results from an inhibition of pollen tube growth. Crosses between alpha'alpha hybrids carrying alpha complexes of different racial origin are compatible. Likewise, crosses of alpharalphas with races, either as male or female parents, produce seed. On the other hand, betabeta hybrids, which also occur, but much less frequently, are perfectly self-fertile. These are briefly the observations which led to the following hypothesis:

Each alpha biennis group 1 complex carries a specific incompatibility allele, $S_{1}, S_{2}, S_{3} \ldots \ldots S_{\mathrm{n}}$. The allele at the homologous locus in the beta complex produces no incompatibility effect and is indicated by the symbol, $S_{\mathrm{f} .}{ }^{2}$ When an alpha. beta, i.e., a complex-heterozygote, is self-pollinated, the pollen carrying the alpha complex cannot develop, since it possesses the same $S$ allele that is present in the stylar tissue. Thus, the $S$ allele acts as a pollen lethal. Only pollen carrying the beta complex with its $S_{f}$ allele can effect fertilizations. If eggs carrying the alpha complex as well as those bearing the beta complex are produced, then beta beta as well as alphabeta combinations should occur among the progeny from selfing. Since betabeta homozygotes are not produced, it is suggested that beta eggs may largely be eliminated through megaspore competition. The origin of the true-breeding translocation heterozygote can thus be explained as the result of a hybridization between two forms differing in segmental arrangement, one of which possessed a self-incompatibility allele system. Once the complex-heterozygote came into being, its balanced lethal system, consisting of incompatibility alleles on the one hand, and megaspore competition on the other, became further reinforced by the accumulation of recessive detrimental and lethal mutations which are protected from segregation by the chromosome ring formation.

On the basis of the incompatibility allele hypothesis it is possible to predict the compatibilities of the progenies of crosses involving biennis group

\footnotetext{
${ }^{2}$ In the earlier paper the allele lacking incompatibility properties was designated by the symbol $(+)$. The $S_{t}$ designation is being adopted to conform with the system used by other workers.
} 
1 races or their hybrids. In the present paper these predictions will be outlined, followed by a report of the results actually obtained in compatibility tests.

MATERIALS AND METHODS.-In the original study (Steiner, 1956) seventeen different biennis group 1 races were crossed in most of the possible combinations. Among the progenies seventy-four different alphaalpha hybrids were obtained. A representative group of these were selected for making crosses of the following types:

(1) Crosses between different alpharalphas;

(2) Crosses between alpha-alphas and biennis group 1 races;

(3) Crosses between alpharalphas and synthesized alphabbetas; ${ }^{3}$

(4) Crosses between races and synthesized alpha betas.

In each cross predictions regarding the nature of the progenies expected were made based on the incompatibility allele hypothesis. In order to obtain again a series of $F_{1}$ alpharalphas which could be used for compatibility tests, a selected group of biennis group 1 races were crossed in all possible combinations. The following races were used, because all are known to transmit the alpha complex through the pollen in outcrosses:

Birch Tree 1
Birch Tree 2
Camp Peary E
Hot Springs
Paducah
Walkerton

Compatibilities of the progenies obtained from all crosses were tested by determining pollen tube growth in the style. Since the alpharalpha hybrids are naturally self-pollinated, it proved desirable to emasculate flowers to be used in the compatibility tests. This was done $24-30 \mathrm{hr}$. before pollination. Twenty-four hr. after pollination the style was removed and fixed in 3 parts absolute alcohol to 1 part acetic acid mixture for at Jeast an hour. It was then placed in a $1: 1$ mixture of 50 per cent alcohol and concentrated $\mathrm{HCl}$ for a few moments, followed by a minute or two successively in each of the following: (1) 70 per cent alcohol-IKI; (2) water; (3) 20 per cent lactic acid-IKI. The style was mounted on a slide in the lactic acid-IKI and flattened with a cover slip. Treatment in the HCl-alcohol mixture makes the style considerably easier to flatten. The lactic acid serves as a clearing agent as well as a convenient

\footnotetext{
${ }^{3}$ A synthesized alpha beta brings together the alpha complex of one race and the beta of a different race; those used here differ from the naturally-occurring alpha . betas only in that they combine complexes from different geographical collections.
}

mounting medium. The slides can be kept for at least six to eight weeks if necessary, without any further processing.

REsults.-(1) Crosses between alphalalphas. -Representative cultures from among these crosses were selected for the compatibility tests. For example:

\section{$\alpha$ Birch Tree $1\left(\mathrm{~S}_{5}\right) \cdot \alpha$ Camp Peary E $\left(\mathrm{S}_{3} \times\right.$ $\alpha$ Birch Tree $1\left(S_{5}\right) \cdot \alpha$ Hot Springs $\left(S_{1}\right)$}

The progeny from this cross should include the two compatibility classes, $S_{1} S_{5}$ and $S_{1} S_{3}$. By crossing members of the progeny to the proper $F_{1}$ alpha - alphas, it should be possible to identify the genotype of each class. Thus, $S_{1} S_{5}$ should be in. compatible with the hybrid, alpha Birch Tree 1 $\left(S_{5}\right) \cdot$ alpha Hot Springs $\left(S_{1}\right)$, but compatible with alpha Hot Springs $\left(S_{1}\right)$ alpha Camp Peary $E\left(S_{3}\right)$. These predictions were confirmed by the compatibility tests. Of twelve plants of this progeny whose compatibilities were determined, four fell into one class, eight into the other. The latter class was incompatible with the $\mathrm{F}_{1}$ hybrid, alpha Birch Tree 1 ( $\left.S_{5}\right)$-alpha Hot Springs $\left(S_{1}\right)$, and thus possessed the genotype, $S_{1} S_{5}$. A total of eight cultures from crosses between alpha-alphas were tested in this way and in every case the results conformed to those expected on the basis of the incompatibility hypothesis.

(2) Crosses between biennis group 1 races and alpharalphas.-The cross, Camp Peary $E\left(S_{3} S_{\mathrm{f}}\right)$ $\times \propto$ Warwick $A\left(S_{6}\right) \cdot \alpha$ Hot Springs $\left(S_{1}\right)$, should produce among the alphaalpha progeny two compatibility classes, $S_{1} S_{3}$ and $S_{3} S_{6}$. These were shown to occur by the compatibility tests. On the other hand, the cross, Camp Peary $E\left(S_{3} S_{\mathrm{f}}\right) \times \alpha$ Paducah $\left(S_{4} \cdot \alpha\right.$ Camp Peary $E\left(S_{3}\right)$, should produce only one compatibility class among the alpha.alpha, alpha Camp Peary $E \quad\left(S_{3}\right) \cdot$ alpha of this class should be incompatible with the $F_{1}$ alpha - alpha, alpha Camp Peary $E\left(S_{3}\right) \cdot$ alpha Paducah $\left(S_{4}\right)$. These predictions were shown to be correct by compatibility tests.

In crosses in which the alphaalphas are hybridized with races, using the latter as male parents, two types of progenies are expected if incompatibility alleles are operative. Thus, when alpha Camp Peary E $\left(S_{3}\right)$ alpha Hot Springs $\left(S_{1}\right)$ is: crossed with Camp Peary $E\left(S_{3} S_{\mathrm{f}}\right)$, only alpha. betas should occur among the progeny since alpha Camp Peary $E$ pollen $\left(S_{3}\right)$ cannot develop in the style of the alpharalpha carrying the same allele. On the other hand, when the alpharalphas used as female parents carry $S$ alleles which differ from that in the alpha complex of the race, alpha. alphas should occur among the progeny. These expectations have been confirmed; in the cross above, only alphabetas appeared among the offspring, while in the following crosses the progeny included alpha-alphas: 
$\alpha$ Friendship $\left(S_{7}\right) \cdot \alpha$ Paducah $\left(S_{4}\right) \times$ Camp Peary E $\left(S_{3} S_{f}\right)$

progeny :

$$
\begin{aligned}
& 2 \text { alpha } \cdot \text { alphas } \\
& 1 \text { alpha } \cdot \text { beta }
\end{aligned}
$$

$\alpha$ Warwick $A\left(\mathrm{~S}_{6}\right) \cdot \alpha$ Hot Springs $\left(\mathrm{S}_{1}\right) \times$ Camp Peary E $\left(S_{3} S_{f}\right)$

progeny :

3 alpha $\cdot$ alphas

46 alpha $\cdot$ betas

Similar results were obtained in six other crosses falling into this category.

(3) Crosses between alphalphas and synthesized alphabetas. - The following are examples of crosses in this category which provide evidence supporting the incompatibility allele hypothesis:

$$
\begin{aligned}
& \alpha \text { Paducah }\left(\mathrm{S}_{4}\right) \cdot \beta \text { Hot Springs }\left(\mathrm{S}_{\mathrm{f}}\right) \times \\
& \alpha \text { Paducah }\left(\mathrm{S}_{4}\right) \cdot \alpha \text { Hot Springs }\left(\mathrm{S}_{1}\right)
\end{aligned}
$$

The progeny consisted of only one compatibility class, namely, $S_{1} S_{4}$, as expected.

$$
\begin{gathered}
\alpha \text { Paducah }\left(S_{4}\right) \cdot \alpha \text { Hot Springs }\left(S_{1}\right) \times \\
\alpha \text { Paducah }\left(S_{4}\right) \cdot \beta \text { Hot Springs }\left(S_{f}\right)
\end{gathered}
$$

The progeny was composed entirely of alpha . betas, as expected, since the alpha pollen carrying the $S_{4}$ allele could not develop.

(4) Crosses between races and synthesized alpha - betas.-The cross, Hot Springs $\left(S_{1} S_{\mathrm{f}}\right) \times$ a Hot Springs $\left(S_{1}\right) \cdot \beta$ Camp Peary $L\left(S_{\mathrm{f}}\right)$, produced only alpha - beta offspring as predicted by the incompatibility allele hypothesis. This result might be interpreted, however, as simply a more effective functioning of a beta complex when competing with an alpha complex. That such an interpretation is unwarranted is shown by the following crosses:

Paducah $\left(\mathrm{S}_{4} \mathrm{~S}_{\mathrm{f}}\right) \times \propto$ Hot Springs $\left(\mathrm{S}_{1}\right)$.

$\beta$ Camp Peary L $\left(\mathrm{S}_{\mathrm{f}}\right)$

$45 / 45$ alpha alphas

Camp Peary E $\left(\mathrm{S}_{3} \mathrm{~S}_{\mathrm{f}}\right) \times$ Hot Springs $\left(\mathrm{S}_{1}\right)$.

$\beta$ Camp Peary L $\left(\mathrm{S}_{\mathrm{f}}\right)$

$37 / 40$ alpha $\cdot$ alphas

Birch Tree $2\left(\mathrm{~S}_{2} \mathrm{~S}_{\mathrm{f}} \times\right.$ Hot Springs $\left(\mathrm{S}_{1}\right)$.

$\beta$ Camp Peary L $\left(\mathrm{S}_{\mathrm{f}}\right)$

$27 / 27$ alpha $\cdot$ alphas

Hopkinsville $\left(\mathrm{S}_{\mathrm{s}} \mathrm{S}_{\mathrm{f}}\right) \times$ Hot Springs $\left(\mathrm{S}_{1}\right) \cdot$

$\beta$ Camp Peary L $\left(S_{\mathrm{f}}\right)$

$4,9 / 49$ alpha $\cdot$ alphas

These crosses emphasize that alpha Hot Springs can function effectively as a pollen complex unless its incompatibility allele is present in the style.

Discussion.-All crosses which have been analyzed show complete consistency botween the results obtained and those expected if incompatibility alleles are operative. It seems reasonable, therefore, to conclude that the alpha biennis group 1 complexes carry incompatibility alleles. In selfpollinations these alleles prevent the functioning of pollen carrying the alpha complex; thus alphaalpha homozygotes cannot occur among the progeny obtained from selfing a complex-heterozygote. In those races which transmit the alpha complex through the pollen in outcrosses this appears to be the only mechanism which eliminates alpha pollen in self-pollinations. In races which in outcrosses rarely or never transmit the alpha complex through the pollen, alpha pollen must be eliminated in some other way, since incompatibility alleles would normally be inoperative in outcrosses. The nature of this second type of pollen lethal is currently under investigation.

No attempt has been made to explain the ratios obtained among the progenies of these crosses. The alpha complexes, being derived from complexheterozygotes. carry lethal and deleterious genes which have been preserved by the genetic mechanism characterizing these forms. Since the alpha - alphas used in these experiments were structurally homozygous, segregation of these genes could occur freely, and undoubtedly affected the ratios of compatibility genotypes that were obtainced. Moreover, the fact that complexes may compete in pollen tube growth or in megaspore development probably makes usual deviations from expected Mendelian ratios. Until these aspects of the genetic mechanism of Oenothera are more thoroughly analyzed, it is unlikely that appreciable sionificance can be attached to the ratios of crosses of the type discussed above.

Another question of fundamental importance concerns the distribution of incompatibility alleles among other phylogenetic groups of complex-heterozygotes. As previously mentioned. the presence of incompatibility alleles in complex-heterozygotes leads to an attractive hypothesis for explaining the origin and evolution of these specialized forms. This hypothesis can obviously be of general significance only if incompatibility alleles can be demonstrated in other groups of complex-heterozygotes, not only within the subgenus Euoenothera, but in other subgenera as well. A survey of the euoenotheras for the presence of incomnatibility alleles has been undertaken; it is hoped that some information regarding the distribution of incompatibility alleles in this subgenus will be available at the end of the next growing season.

\section{SUMMARY}

Additional evidence of a self-incompatibility 
allele mechanism in the biennis 1 phylogenetic grouping of the subgenus Euoenothera is presented. Compatibility classes among the offspring of a series of crosses involving biennis group 1 races and their hybrids were determined. Without excep- tion the results obtained confirmed those expected according to the incompatibility allele hypothesis.

Department of Botany and the Botanical Gardens, University of Michigan, Ann Arbor, Michican

\section{I.ITERATURE CITED}

Crowe, Lescie K. 1956. The evolution of incompatibility in species of Oenothera. Heredity 9: 293-322.

EMERson, S. 1938. The genetics of self-incompatibility in Oenothera organensis. Genetics 23: 190-202.

1939. A preliminary survey of the Oenothera organensis population. Cenetics 24: 524-537.

LEWIS, D. 1947. Competition and dominance of incompatibility alleles in diploid pollen. Heredity 1 : 85108.
1948. Structure of the incompatibility gene. I. Spontaneous mutation rate. Heredity 2: 219-236. 1949. Structure of the incompatibility gene. II. Induced mutation rate. Heredity $3: 339-355$.

1951. Structure of the incompatibility gene. III. Types of spontaneous and induced mutation. Heredity 5: 399-414.

Steinels, ERich. 1956. New aspects of the balanced lethal mechanism in Oenothera. Genetics 41: 486-500.

\section{PERSISTENT NUCLEOLI AND GRASS SYSTEMATICS ${ }^{1}$}

\section{Walter V. Brown and William H. P. Emery}

IT is Isuali.y stated that the nucleolus disapjears at late prophase of mitosis (Gates, 1942; Vincent, 1955). There is considerable evidence, however, that it is not unusual for plant nucleoli to persist to mitotic metaphase or later. Frew and Bowen (1929) cited the presence of nucleoli at metaphase and often at anaphase in 11 genera of monocotyledons and dicotyledons. Zirkle (1928) reported a similar condition in maize, the nucleoli heing revealed by the use of a basic fixative. Ramanujam (1938) detected nucleoli persisting to telophase in root-tip cells of one strain of rice. More recently $T$ jio (1948) described the peculiarilies of persistent nucleoli in Ceiba pentandra, D'Amato Avanzi (1953) found persistant nucleoli in 16 species of Cassia (all the species investigated), and Gori (1956) reported them in 10 species of Reseda.

It was reported by Zirkle, Ramanujam, and $\mathrm{Tj}$ io that at times the nucleolus is included on the metaphase plate, and when this occurs some force appears to "squeeze" it so that part moves toward one spindle pole and part toward the other. The movement in both directions at the same time produces a dumbbell-shape. Later, but still at metaphase, it separates into two parts that continue movement to the spindle poles. There are other cells in which the nucleolus is not included in the metaphase plate but is, nevertheless, contained within the spindle. It then moves as a whole to one pole. At late metaphase, therefore, one of three conditions may prevail: there may be (1) one nucleolus at one pole, (2) one at each pole, or (3) no evident nucleoli. Ramanujam stated that nucleoli that persist to telophase are not included within the reforming telophase nuclei. Rather, they are excluded and eventually disintegrate in the cytoplasm.

\footnotetext{
${ }^{1}$ Received for publication Nov. 23, 1956.
}

Materials and methods.-The special fixation and staining method used in this study was that of Rattenbury and Serra (1952). Root tips were fixed in alcohol-formalin-acetic acid $(30: 15: 1$ by volume) for 6-12 hr., hydrolyzed for 1-2 hr., in normal HCl at $60^{\circ} \mathrm{C}$., and squashed in aceto-carmine. This treatment results in very poor staining of chromosomes but good staining of nucleoli.

Plants representing 45 species in 39 genera in 20 tribes of the Gramineae provided material for the study. Most plants were grown in the greenhouse and materials collected during January and February. The selection of species was in part determined by material available in the greenhouse or out of doors. An effort was made to select species that represented as many tribes as possible. Root tips were taken from plants of at least one species in each of 20 tribes, 5 tribes of Festucoideae, 9 tribes of Panicoideae, and 6 tribes that are often classified outside of these two subfamilies (Avdulov, 1931) or appended to them with reservations (Prat, 19:36).

The tribes as recognized here do not correspond to those of Hitchcock (1950) which is a manual for identification of genera and species. Rather, treatments such as those of Prat (1936) and Hub. bard (1948) have been followed for reasons discussed by Stebbins (1956).

Most of the species examined constitute part of the present day flora of Central Texas and are either native or introduced, cultivated or wild. Others are from the Plant Research Institute grass nursery. All plants were identified by the senior author. Voucher specimens of the less common introduced species have been deposited in the herbarium of the University of Texas.

REsulTs.-The sequence of stages reported by Zirkle and Ramanujam were clearly seen in all 